\title{
Anmerkung zur Kontroverse Poulantzas-Miliband
}

Die Kontroverse $z$ wischen Miliband ${ }^{1}$ und Poulantzas ist zum einen die Auseinandersetzung zwischen zwei Forschungsstrategien, zum anderen die zwischen zwei methodischen Ansätzen. Während Miliband sich auf die wKritik herrschenden Ideologien auf der Ebene ihrer eigenen Kategorien beschränke und damit auf Grund fehlender kritischer Begrifflichkeit das durch das zu Kritisierende vorgegebene kategoriale System reproduziert, sucht Poulantzas über die immanente Ideologiekritik hinaus eine theoretische Interpretationsalternative zur herrschenden Ideologie zu entwickeln². Dabei setzt er sich auch gegen die methodologischen Prämissen Milibands ab, mit denen dieser aus einer analytischen Reduktion auf Motivationsanalyse und auf das Betonen interpersoneller Momente das kapitalistische System auf entscheidungs- und handlungstheoretische Elemente, somit die Klassenanalyse auf Elitetheorie, auf handelnde Individuen und Gruppen reduziert, ohne wie Poulantzas die motivationalen und intentionalen Elemente strukturell/funktionell in den Zusammenhang der widersprüchlichen Konstitution und Entwicklung des Kapitalismus rücken und Klassen als (schlußendlich auch personell sich ausdrückende) Substrate der objektiven, sstrukturellen « Bedingungen der kapitalistischen Produktionsweise definieren zu können.

Am Problem der Funktion der Staatsbürokratie ${ }^{3}$ kann dieser Unterschied - wie

1 Vgl. zu Miliband auch die Rezension von Ritsert in diesem Heft auf S. 22I ff. Miliband selbst definiert sich in der Tradition von $C$. Wrighe Mills, dessen einschlägige Arbeiten ähnlich problematisch sind wie die Milibands. Vgl. insbesondere C. Wright Mills, The Power Elite, New York 1956. Eine neuere, informative Variante dieses Erklärungsmusters findet sich bei: G. William Domhoff, Who Rules America? Englexood Cliffs x967; ders., The Higher Circles, New York r 971 (Vintage).

2 Vgl. zu dessen präziserer Entwicklung: Nicos Poulantzas, Pouvoir politique et classes sociales, Paris $1970^{2}, 398 \mathrm{~S}$. (Maspero).

3 Diskussionsansätze im Bereich der Analyse der Funktionen bundesdeutscher Staatsbürokratie sind u. a.:

Joachim Hirsch, Funktionsveränderungen der Staatsverwaltung in spätkapitalistischen Industriegesellschaften, in: Blätter für deutsche und internationale Politik 1969, S. I50-164; ders., Wissenschaftlich-technischer Fortschritt und politisches System, Frankfurt 1970, $293 \mathrm{~S}$. (Suhrikamp), insbes. Kap. I \& IV.

Joachim Hirsch / Stephan Leibfried, Wissenschaftspolitische Materialien (Abschnitr D: Verwaltungsforschung), Frankfurt 5971.

Claus Offe, Politische Herrschaft und Klassenstrukturen. Zur Analyse spätkapitalistischer Geselischaftssysteme, in: Kress/Senghaas (Hg.), Politikwissenschaft. Eine Einführung in ihre Probleme, Frankfurt r969, S. Iss-r\$g (EVA); ders./Wolf Dieter Narr, Einleitung zu dem Sammelband: Woblfahrtsstaat und Massenloyalität, Köln $197 x$ (Kiepenheuer/Witsch).

Ulrich Karl Preuß, Neuere Tendenzen der Organisation der Staatsverwaltung, verv. Man. Mai $1970,42 \mathrm{~S}$.

Zur Theorie des staatsmonopolistischen Kapitalismus, Berlin 1967 , 35s S. (Akademie Verlag); Herbert Meißner, Hg., Bürgerliche Okonomie in modernen Kapitalismus, Berlin 1967, $746 \mathrm{~S}$. (Dietz Verlag); Imperialismus heute. Der staatsmonopolistische Kapitalismus in Westdeutschland, Berlin r 967,823 S. (Dietz Verlag). 
die unten abgedruckten Texte zeigen - schlaglichtartig erhellt werden. Einen nach Miliband gesellschaftliche Herkunft (Rekrutierung) und interpersonelle Bezichungen den Staatsapparat, so leitet Poulantzas die Einheit aus der Funktion ab, die der Staatsbürokratie in der kapitalistischen Klassengesellschaft zukommt, ohne jedoch in der Tradition des Okonomismus der II. und III. Internationalet die Beziehungen zwischen Staatsapparat und herrschender Klasse (oder wie Poulantzas mit Vorliebe formulicrt: herrschenden Klassen) mechanisch kurzzuschließen.

Gegen den empiristischen Reduktionismus Milibandscher Prägung und dic Konzeption vom "Staatsmonopolkapitalismus "s ${ }^{5}$, die in einer Art Verschwörertheorie den Staatsapparat von den Monopolen okkupiert (quasi physiscl in dic Ecke gedrängt) sieht und damit die prinzipiell für möglich erachtete, freilich fundamental andere Rolle des Staatsapparats bei einer nun auch von der KPF von oben, von der Ubernahme der Staatsgewalt ausgchenden Revolution beschränkt, konturiert Poulantzas sein Konzept vom kapitalistischen Staat als Zentrum der politischen Herrschaft der herrschenden Klasse.

In der schlichten Negation dieser beiden Positionen tritt jedoch die Aufgabe der Staatsbürokratie, die Verwertungs- und Realisierungsschwierigkeiten des Kapitals administrativ zu »beheben«, ebenso wie die damit zusammenhängende strategische Bedeutung der Rckrutierungsprozesse der Staatsbürokratien, völlig zurück hinter der Funktion des Staates, Unterschiede unter den einen wherrschenden Block " (bloc au pouvoir) bildenden Klassenfraktionen ${ }^{6}$ einzuebnen und das System gegen es gefährdende Angriffe abzusichern. Ahnlich wie er die Klassen nicht vornehmlich aus ihrer Stellung in den Produktionsverhältnissen definiert, sondern auf dem norwendigen politisch-ideologischen Niederschlag (effet pertinent) ihrer ösonomischen Stellung insistiert ${ }^{7}$, versteht Poulantzas den kapitalistischen Staat nicht primär als »ideellen Gesamtkapitalisten«, sondern als Systembewahrer.

Um diese seine Aufgabe zu erfüllen, die politische Hegemonie der herrschenden Klassen abzusichern, muß - so Poulantzas - der Staar über eine relative Auronomie auch gegenüber den herrschenden Klassen selbst verfügen. Erst dicse ermöglicht es ihm, die Unterschiede unter den herrschenden Klassenf́raktionen zu beseitigen, aus Gründen der Systemerhaltung selbst ökonomische Interessen dieser Klassen zu tangieren und schließlich die Loyalität der Massen dadurchi zu binden, daß er ihnen trügerisch als neuirale Instanz erscheine ${ }^{3}$. Indem Poulantzas die kapitalistische Gesellschaftsformation in verschiedene Ebenen

- Die entsprechenden Auseinandersctzungen von Poulantzas mit dem Okonomismus, zuf deren Hincergrund dessen Theoriekonzeption erst ihr Profil gewinnt, sind leicer bei dem hier gekürzten Nachdruck nicht ausreichend zum Tragen gekommen. Die Arbert von Poulantzas: Fascisme et dictantre. La troisième internationale face au fascime, Paris 1970,402 S. (Maspero) widmet sich hauptsächlich diesem Thema.

5 Vgl. zu dieser Theorievariante den letztgenannten Diskussionsansatz in Fußnote 3.

- Mir den Kategorien Poulantzas' gehe Andrk Gorz an scine Analyse der Widersprüche innerhalb der französischen Bourgeossie heran und leitet die Einheit der traditionellea Bourgeoisicfraktionen und ihren Widerstand gegen Unternehmen der modernistischen und monopolistischen Kapitalfraktion vornelumlich - wie auch Poulantzas - aus den im Klasscnkampf ausgebildeten konservativen Reflexen ab. Val. Gorz, Rétorme et Révolution, Paris 1969 Einlcitung (Deutsch: Die Aktualitär der Revolution, Frankfure r 970 (EVA)).

7 Vgl. Pouvoir politiques er classes sociales a. 2. O. S. 57-109.

8 Die Klassenherrschaft stabilisierende und verscilleiernde Funlction einer sich von der Staatsgewalt absetzenden Branche des Staatsapparats haben am Beispicl des Erzichungssystems Frankreichs Bourdieu und Passeron dargestellt. Cí. Pierre Bourdieu/Jean Claude Passeron, La Reproduction. Elements d'une Théorie du système d'enseignement, Paris I 970 (Maspero), insbes. S. $202 \mathrm{ff}$. 
und Instanzen untergliederr - das „Politische«, „Okonomischex, „Ideologische«... -, die zwar funktional verschränkt, aber zugleich auch relativ autonom scien, gelinge es ihm, die herrschaftsstabilisierende und -verschleiernde Funktion des Staates herauszuarbeiten; aber er kann begrifflich die Möglichkeiten und Grenzen staatlicher Intervention nur vermittelt als politische, nicht aber durch die Kapitalbewegung selbst geserzte erfassen.

Generell scheint es, als ginge Poulantzas in seiner Analyse eher vom Bild des Konkurrenz- als des Monopolkapitalismus aus, denn er berücksichtigt kaum die erst neuerdings wieder von Narr und Offe betonte "Steuerungs\&funktion des kapitalistischen Staats im kapitalistischen Reproduktions- und Produktionsprozeß. Poulantzas beschränkt das Krisenmanagement des kapitalistischen Staates vornehmlich auf die Abwehr systemgefährdender Angriffe, die vom Proletariat ausgehen und die die repressiven und ideologischen Apparate auffangen beziehungsweise vermeiden sollen. Die Eingriffe des Staates, die darauf zielen, z. B. durch die Organisarion des Marktes die Realisierungsschwierigkeiten der Einzelkapitale zu vermindern oder durch die Förderung wissenschaftich-technischer Innovation die Kapitalverwertung zu begünstigen und den Verwcrtungsschwierigkeiten staatsinterventionistisch zu begegnen, treten in den gesamten theoretischen Arbeiten Poulantzas' hinter den politischen Imperativ der Absicherung der politischen und sozialen Struktur des Kapitalismus zurück. Die das gegenwärtige Stadium der kapitalistischen Entwicklung kennzeichnenden funktionell imperativischen Beziehungsverhältnisse von »politischen und *ökonomischen « Funktionen der Staatsbürokratien verkürzt Poulantzas auf deren (auch bestehende!) Aufgabe im Klasscnkampf, wie dieser überhaupe die generierende Matrix seiner Theoriebildung ist: Le caractére même d'une tendance historique, Marx l'avait souligné, est commandé précisément, et en dernière analyse, par le fait que le procès économique est surdéterminé par la lutte des Classes, qui détient le primat « ${ }^{10}$.

Die Erfahrungen der französischen Klassenkämpfe ebenso wie die theoretische Frontstellung Poulantzas' gegen den Okonomismus der KPF, der Material für die Grenzen, nicht jedoch für die Mögliclikeiten revolutionärer Praxis liefert, erklären diesen Bezugsrahmen; jedoch greift Poulantzas mit diesem analytischen Instrumentarium zu kurz.

Durch die Polemik gegen die phänomenologischen Ausarbeitungen, die Miliband übcr die Relsrutierungsprozesse des Staatsapparats vorlegt, fällt Poulantzas jedoch nur in das genaue Gegenteil und vernachlässigt sowohl die analytische Bedeutung, die die klassenspezifische Rekrutierungsgeschichte der Staatsbürokraten für die Staatsfunktionen haben kann, als auch deren je nach Klassenkonstellation unterschiedliche funktionelle Entscheidungskomperenz, deren intern differenzierte, je unterschiedliche Organisation von Widersprüchen als zu unterdrückende, zu verdrängende, zu vertagende ... Freilich liefert Poulantzas zum ersten Aspekt in »Fascisme et Dictature* selbst eine Korrektur, wenn er die Grenzen der staatsinterventionistischen Tätigkeit zugunsten des Kapitals im Deurschland Bismarclss auf das Monopol der Staatsbürokratie zurückführr, das ein feudal tingierter Adel inne hatte. Man vermißr jedoch eine Gewichtung der - relativen Autonomie der Staatsbürokratie je nach der strategischen Bedeutung, die die einzelnen Teile des Apparats in der Politik des Kapitals annehmen. Dabei wäre zwischen den repressiven Instanzen, beispielsweise, die das allge-

\footnotetext{
- Spärkapitalismus, Manuskript, S. toff. (s. Fußnote 3).

10 Fascisme et dictature a. 2. O. S. 39.
} 
meine Interesse des Gesamtkapitals durchsetzen, und jenen zu unterscheiden, in denen qua Realisierung administrativer Steucrungsfunktionen verschiedene Kapitalfraktionen zusammenstoßen, und dann auch Rekruticrungsprozesse einen spezifischen Erkenntniswert haben.

Schließlich sei zur Einschätzung der Struktur der Theorie von Poulantzas darauf hingewiesen, $\mathrm{da} B$ er eine eigentümliche $Z$ wischenposition $z$ wischen marxistischer Theorie einerseits und dem, was in der Bundesrepublik unter der Bezeichnung $*$ Systemtheorie* firmiert, andererseits einnimmt. Der letzteren Theorievariante zufolge (die parallel zu Poulantzas personalistische Erklärungsansätze qua Theorie der Handlungssysteme ausschließt und so theoretisch Elitetheorien negiert) ist der Staat anzusehen als $»$ Ausbildung der politischen Sphäre zu relativer gesellschaftlicher Autonomie, als Untersystem der Gesellschaft, das eine spezifische Funktion in der Gesamtordnung erfüllt und dafür ausdifferenziert und freigestellt ist « ${ }^{11}$. Eint beide Ansätze das Konzept relativer Autonomie samt einer Ausdifferenzierungsthese, so trennt sie die Definition der Struktur des Bezugsproblems: für Luhmann die »Reduktion von Komplexität«, die Bestandserhaltung des Systems gegenüber einer kontingenten Welt; für Poulantzas die ideologische und politische Reduktion von Komplexität, die sich aus der Bestandserhaltung eines Systems der Klassenherrschaft ergibr. Beide Theorien teilen auch eine spezifische Dethematisierung: den Staatsapparat in seinen spczifisch kapitalverwertungsrelevanten Aktivitäten voil zu ignorieren und dann auch insgesamt eine eigentümliche Unabgeleitetheit der Analysen aus einer Kritik der politischen Okonomie mit der Folge, daB beide Theorieansätze, wenn auch auf - politisch recht relevantem - unterschiedlichem Erkenntnisniveau, in ihrer Begrifflichkeit als reifiziert, ontologisiert erscheinen.

Heinz Gerbard Haupt Stephan Leibfried

"Niklas Luhmann, Grundrechte als Institution. Ein Beitrag zur politischen Soziologie, Berlin $1965,5.30$. Leider ist dieser Theorieansatz im gesamten Schrifttum (einschließlich der Kritischen Justiz) bislang weder adäquat behandelt (= kritisiert) noch demgemäß rezensiert worden. In dieser Hinsicht führt auch diese jerze veröffentlichte Auscinandersetzung zwischen Luhmann und Habermas kaum weiter. Vgl. Jürgen Habermas / Niklas Luhnann, Theorie der Gesellschait oder Sozialtechnologie - Was leister die Systemforschung? Frankfurt I975. Luhmanns Theorie wird hier nuche im Zusammenhang einer spezifischen historischen Entwicklungsstufe des Kapitalismus, ihrer dazu spezifischen (Theorie-strukturell bedingten) Dethematisierungen untersucht, sondern auf Luhmann gleicher, abstrakter (sprachtheoretischer, sprachphilosophischer, methodologischer...) Ebene. Dadurch veriiert diese Arbeit jeden politısch-analytaschen Wert und erhält einen esoteriscinen Clıarakter; dies findet seınen AusCruck auch in der personalisierenden Form der Auseinandersetzung, als einer zwisehen "gebilćete(n) und überraschende(n) Geiste(rn) $<$ (S. I45; etwas vertiremdet). 